\title{
Application of tissue microarrays for receptor immunohistochemistry in breast carcinoma
}

\author{
Anna Glajcar, Karolina Kaczmarczyk, Joanna Szpor, Krzysztof Okon
}

\author{
Department of Pathomorphology, Jagiellonian University, Collegium Medicum, Cracow, Poland
}

\begin{abstract}
The current treatment of breast cancer, the most frequent malignancy found in females, requires the study of biomarkers. The standard set of these includes at least an estrogen receptor, a progesterone receptor and a HER2 receptor, although many other factors have been shown to contribute to the prognosis. Tissue microarrays have been introduced to decrease costs and workload of immunohistochemistry applied to large collections of samples. The aim of the study was to test the performance of this technology on three basic biomarkers of breast carcinoma in 106 cases of invasive breast carcinoma. Tissue microarrays composed of 3 cores sized $0.6 \mathrm{~mm}$ per case were constructed and stained by standard immunohistochemistry. The results were assessed on virtual slides created with an Aperio scanner. A sensitivity and specificity of 0.83 and 0.88 was obtained for the estrogen receptor, 0.76 and 0.88 for the progesterone receptor, 0.69 and 0.96 for HER2. In conclusion, TMA technology may give results comparable to the diagnosis based on whole sections, and the clinicopathologic correlations for the immunohistochemistry performed by both methods are fairy similar. (Folia Histochemica et Cytobiologica 2013, Vol. 51, No. 4, 326-332)
\end{abstract}

Key words: breast cancer; tissue microarrays; prognostic factors; ER; PR; HER2

\section{Introduction}

As the most frequent cancer occurring in females, breast carcinoma (BC) is a disease of an outstanding importance both for clinician and pathologist. Although the primary classification is done by standard histopathology, immunohistochemistry is increasingly used both for classification and prognosis. The routinely used immunohistochemical (IHC) methods include determination of receptor status, namely estrogen receptor (ER), progesterone receptor (PR) and HER2. These define three main classes of BC: steroid receptor dependent (ER +, PR +, HER2-), HER2 dependent (ER-, PR-, HER2+) and triple negative (ER-, PR-, HER2-). These categories differ in their aggressiveness as well as treatment. Many other biomarkers were also shown to contribute to the prognosis, and some are on the way to become diagnostic standards [1-3]. Currently, immunohistochemistry for ER, PR and

Correspondence address: K. Okon, Chair of Pathomorphology, Jagiellonian University, Collegium Medicum,

Grzegorzecka St. 16, 31-531 Krakow, Poland;

e-mail: mpokon@cyf-kr.edu.pl
HER2 should be performed in every case of BC; the side effects in the diagnostic therapeutic improvement are the increased costs of histological examination.

Tissue microarrays (TMA) are commonly used to reduce costs and efficacy of biomarker assessment. This method combines material from several tissue blocks into a single recipient block, reducing the costs and labor considerably, however, at the expense of lower representativeness. Some authors proposed the use of TMAs for routine IHC classification of BC while others advocated more caution in this regard [4-7]. Since our lab has specific experience in using the TMA method both for immunohistochemistry and in situ hybridization, we decided to test the feasibility of TMA assessment of receptor status in BC and compare them with the results obtained by routine immunohistochemistry. We wanted also to see how many cases would be lost from the TMA assay, and how this loss would influence overall results of the receptor assays.

\section{Material and methods}

The material consisted of 106 unselected archival cases of invasive ductal breast carcinoma. The clinicopathologic 
Table 1. Clinicopathologic data of the cases under study

\begin{tabular}{|c|c|c|}
\hline Age & \multicolumn{2}{|c|}{ Mean 56.3 years (range 28 to 85, SD 14.16) } \\
\hline \multirow[t]{5}{*}{ Stage } & & No $(\%)$ \\
\hline & pT1a & $8(7.55 \%)$ \\
\hline & pT1b & $53(50 \%)$ \\
\hline & pT1c & $41(38.68 \%)$ \\
\hline & pT4b & $4(3.77 \%)$ \\
\hline \multirow[t]{6}{*}{ Lymph node status } & & No $(\%)$ \\
\hline & No & $61(57.55 \%)$ \\
\hline & N1mi & $6(5.66 \%)$ \\
\hline & $\mathrm{N} 1 \mathrm{a}$ & $26(24.53 \%)$ \\
\hline & $\mathrm{N} 2 \mathrm{a}$ & $5(4.72 \%)$ \\
\hline & $\mathrm{N} 3 \mathrm{a}$ & $8(7.55 \%)$ \\
\hline \multirow[t]{4}{*}{ Grade } & & No $(\%)$ \\
\hline & G1 & $17(16.04 \%)$ \\
\hline & G2 & $54(50.94 \%)$ \\
\hline & G3 & $35(33.02)$ \\
\hline
\end{tabular}

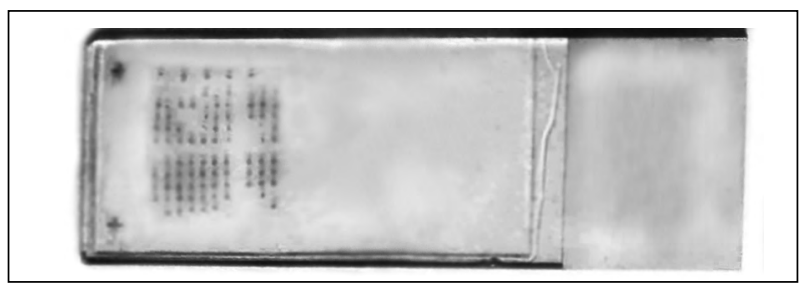

Figure 1. One of the routine tissue microarray slides used in the study. Notice the asymmetric arrangement of individual cores, necessary for proper orientation. Also, the loss of some cores may be appreciated

data is shown in Table 1. The hematoxylin and eosin stained slides were evaluated microscopically and the ones containing areas of best-preserved cancer tissue were chosen for TMA construction. The source area was marked on the slide, copied onto paraffin block and tissue cores were transferred into recipient block. The TMAs were constructed using MTA1 device (Beecher Instruments, Sun Prairie, WI, USA) (Figure 1). From each case, 3 cores sized $0.6 \mathrm{~mm}$ were extracted. From the blocks, $2 \mu \mathrm{m}$ sections were prepared and used for immunohistochemistry. The immunohistochemistry for ER and PR was done by routine manual method. Briefly, the slides were de-waxed, rehydrated and incubated in $3 \%$ peroxide solution for 10 minutes to block the endogenous peroxidase activity. Antigen retrieval was carried out by heating in citrate buffer for 30 minutes. The Lab-Vision detection system was used. Staining for HER2 was done by automatic method using the Benchmark Classic device (Ventana Medical Systems, Tucson, AZ, USA), according to the manufacturers' protocol. The staining results are shown on Figures 2, 3 and

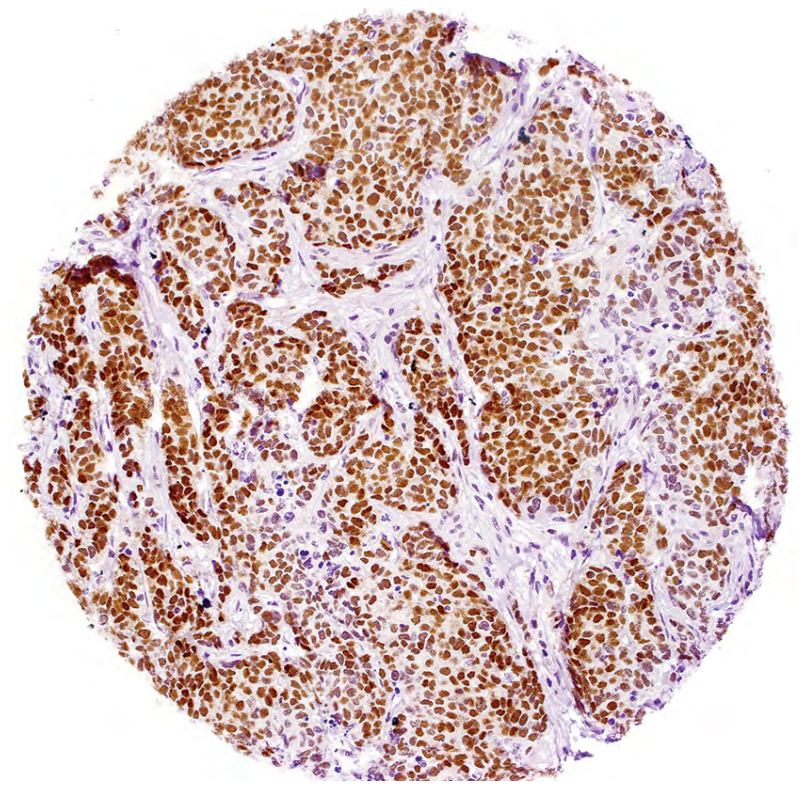

Figure 2. Strong and uniform nuclear reactivity for progesterone receptor. Original magnification $200 \times$

4. Primary antibodies used for the study were for estrogen receptor EMR02 (Leica Biosystems, Wetzlar, Germany), for progesterone receptor SAN27 (Leica Biosystems, Wetzlar, Germany) and for HER2 4B5 (Ventana Medical Systems, Tucson, AZ, USA).

The slides were scanned with Aperio CS device (Aperio Inc., Vista, CA, USA) and virtual slides visually reviewed and scored. For the evaluation of estrogen and progesterone receptors, the percentage of strongly positively stained nuclei was assessed. For HER2 assessment, the cores were classified into the standard categories according to ASCO/CAP guidelines [8]. Cases 0 and $1+$ on HER 2 are refferend to as 'negative', cases $2+$ as 'ambigous', and 3+ as 'positive'. This is in concordance with the clinical meaning of the HER2 assay: in $3+$ cases anti-HER2 antibodies are used for treatment, $2+$ cases require in situ hybridisation before therapy choice, while 0 and $1+$ cases are treated by other methods. The results of standard (whole sections) immunohistochemistry performed manually were obtained from the department's files.

Statistical analysis. The statistical analysis was done using Excel 2010 (Microsoft, Redmond, WA, USA), Statistica 10 (StatSoft, Tulsa, OK, USA) and QuickCalcs (GraphPad Software Inc., La Jolla, CA, USA http://graphpad.com/quickcalcs/) programs. Pearson's $\chi^{2}$, Mann-Whithey U test, kappa statistics were used, as deemed appropriate. Bland-Altman analysis was performed with Statistica software with a macro written by Matt Coates, available from http://sdn.statsoft. com/STATISTICAVisualBasicExamples/BlandAltmanPlot. aspx. The correlation between variables was assessed by Spearman's correlation coefficient. The significance level was set to $P<0.05$. 


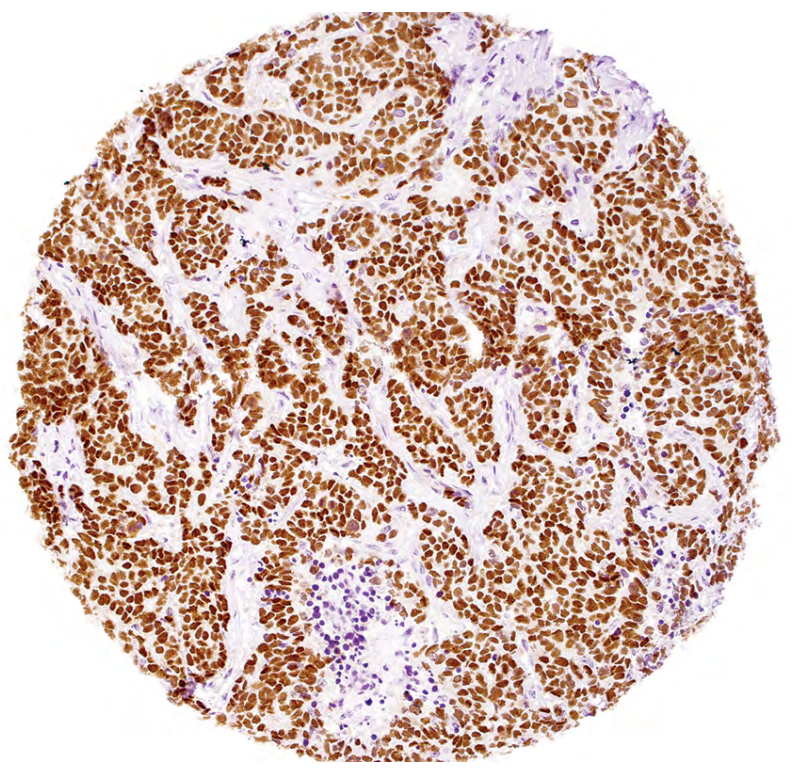

Figure 3. Infiltrating ductal carcinoma positive for estrogen receptor; majority of cells are positive, yet the reaction is weaker than on Figure 2. Original magnification $200 \times$

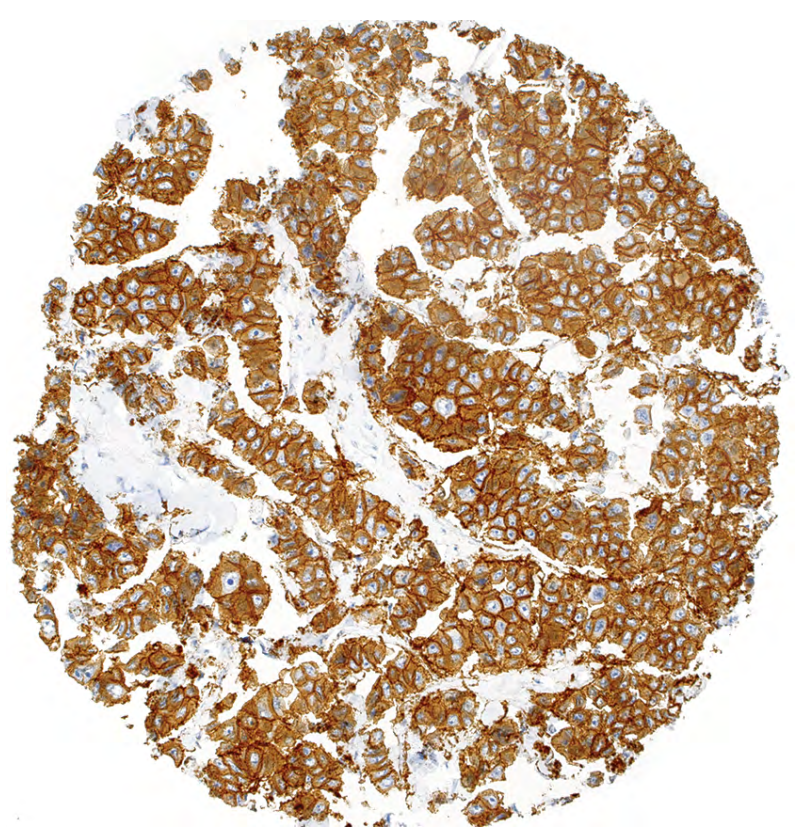

Figure 4. Strong reactivity for HER2 receptor. Such tumor is likely to show good response to HER2 targeted treatment. Original magnification $200 \times$

\section{Results}

On whole-slide immunohistochemistry which had been performed manually, the average percentage of ER positive cells was $68.61 \%$ (range 0 to 100 , SD 36.65). The average percentage of $\mathrm{PR}$ positive cells was $60.45 \%$ (0 to 100, 39.98: range and SD, respectively). HER2

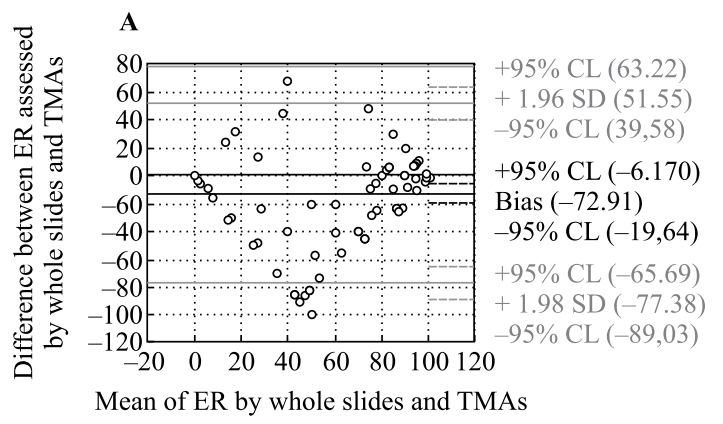

B
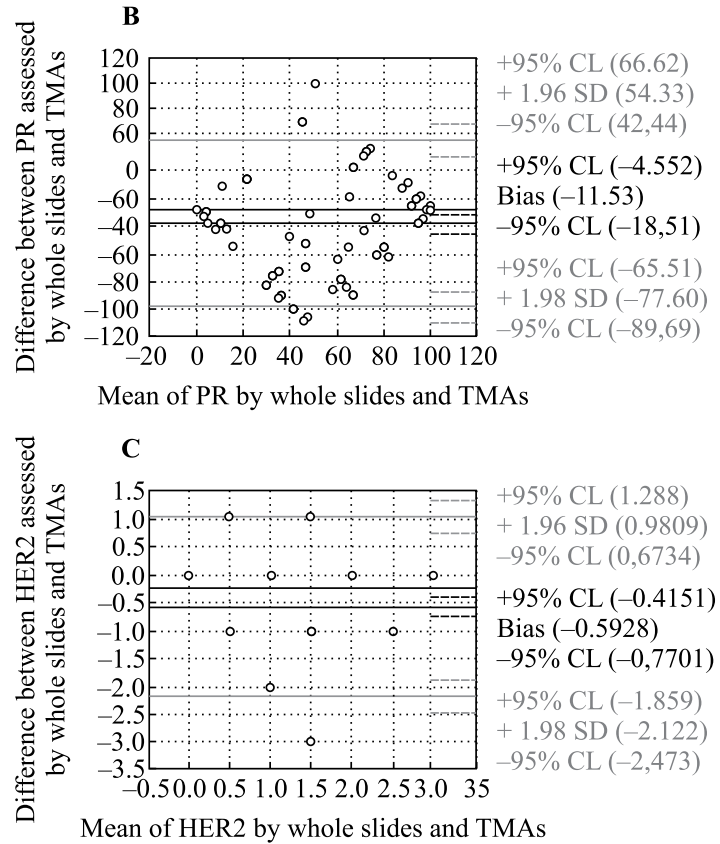

Figure 5. Bland Altman plots of the estrogen receptor (A), progesterone receptor (B) and HER2 (C) assessed by whole slides and tissue microarrays

was 0 in 11 cases $(10.38 \%), 1+$ in 53 cases $(50 \%)$, $2+$ in 28 cases $(26.42 \%), 3+$ in 14 cases $(13.21 \%)$.

According to standard threshold, 16 cases $(15.09 \%)$ were ER negative and 90 cases $(84.91 \%)$ were ER positive; 27 cases $(25.47 \%)$ were PR negative and 79 cases $(74.53 \%$ ) were PR positive; 64 cases (60.38\%) were HER2-negative, 28 cases $(26.42 \%)$ were HER2 ambiguous (requiring FISH), 14 cases (13.21\%) were HER2-positive.

On TMA immunohistochemistry, the average percentage of ER positive cells was $53.56 \%$ (range 0 to 100, SD 42.57$)$; however, $12(11.32 \%)$ cases were lost from TMA in processing. The average percentage of PR positive cells was $37.05 \%$ (range 0 to 100 SD 41.78); 14 (13.2\%) cases were lost from TMA in processing. HER2 was 0 in 39 cases $(36.79 \%), 1+$ in 21 cases $(19.81 \%), 2+$ in 12 cases $(11.32 \%), 3+$ in 9 cases ( $8.49 \%$ ); 25 cases $(23.58 \%$ ) were lost from the TMA. According to the standard threshold, 27 cases 
$(25.47 \%)$ were ER negative and 67 cases $(63.21 \%)$ were ER positive; 39 cases $(36.79 \%)$ were PR negative and 53 cases $(50 \%)$ were PR positive; 60 cases (56.60\%) were HER2 negative, 12 cases $(11.32 \%)$ were HER2 ambiguous (requiring FISH), 9 cases (8.49\%) were HER2 positive.

The results of whole-slide and TMA assessment strongly correlated (for ER $\mathrm{r}=0.66 P<0.001$; for $\mathrm{PR} r=0.73 P<0.001$; for HER $2 r=0.62 P<0.001$ ). However, the results obtained by both methods were not identical. The mean difference for ER was $12.91 \%$ (range -68 to 100, SD 32.89), for PR $11.53 \%$ (range -99 to 90 SD 33.71), and for HER2 $0.59 \%$ (range -1 to 3, SD 0.8). Tables 1 to 3 and Figure 5 show the relationships between clinically relevant receptor immunoreactivities assessed in standard, manually stained histologic slides and TMAs. It is evident that use of TMA has high positive predictive value specificity yet lower negative predictive value and sensitivity. There was no difference in number of available cores between concordant and discordant cases.

The ER, PR and HER2 status did not show significant differences between $\mathrm{pT}$ stages, both in case of whole-slide and TMA immunohistochemistry (data not shown). As might have been expected, there was a statistically significant relationship between the grade of the tumor and receptor status: higher grade cancers tended to be ER and PR negative and HER2 positive more often than the lower grade. These relationships were similar for both whole-slide and TMA immunohistochemistry (Figure 6).

\section{Discussion}

The study of biomarkers considerably increases the effectiveness of cancer prognosis and treatment. Often the multivariate analysis of biomarkers and morphologic features offers quite good discrimination between groups; however, in the routine diagnostic workup, the principal limit is the increased cost and workload. The tissue microarray technology, introduced in the `90s, permitted the analysis of several cases within a single tissue block $[9,10]$. The advantage of this approach is a dramatic reduction in expenses and laboratory workload. In fact, a single TMA may contain as many as hundreds of samples. This may result in a significant reduction of overall costs, especially if many markers are studied within a single experiment. IHC analysis of datasets containing thousands of cases is otherwise impossible or, at best, extremely difficult. Utilizing this method Tamini et al. [11] performed TMA analysis of a large cohort of over $3000 \mathrm{BC}$ cases with sections stained for ER, PR, HER2, CK5/6 and EGFR. These authors used $30.6 \mathrm{~mm}$ cores for each
Table 2. Estrogen receptor positive and negative results assessed by two methods

\begin{tabular}{|l|l|l|l|}
\hline \multicolumn{2}{|c|}{} & \multicolumn{2}{l|}{ TMA } \\
\cline { 3 - 4 } \multicolumn{2}{|l|}{ Whole slides } & Negative & Positive \\
\cline { 3 - 4 } & Positive & 13 & 2 \\
\hline Positive predictive value & 14 & 65 \\
\hline Negative predictive value & \\
\hline Sensitivity & & 0.97 \\
\hline Specificity & 0.52 \\
\hline Kappa & 0.83 \\
\hline
\end{tabular}

Table 3. Progesterone receptor positive and negative results assessed by two methods

\begin{tabular}{|l|l|l|l|}
\hline \multicolumn{2}{|c|}{} & \multicolumn{1}{l}{ TMA } \\
\cline { 3 - 4 } \multicolumn{2}{|c|}{} & Negative & Positive \\
\hline \multirow{3}{*}{ Whole slides } & Negative & 23 & 3 \\
\cline { 2 - 4 } & Positive & 16 & 50 \\
\hline Positive predictive value & 0.94 \\
\hline Negative predictive value & 0.59 \\
\hline Sensitivity & 0.76 \\
\hline Specificity & 0.88 \\
\hline Kappa & 0.56 \\
\hline
\end{tabular}

case and reported to have used 23 TMA blocks. This results in a total of only 115 slides stained $(23 \times 5=115)$, while with standard technology, 15465 slides $(3093 \times 5=$ $=15465$ ) would be stained, a workload that would be prohibitive. As the result, the authors were able to reproduce molecular classification of the $\mathrm{BC}$ cases. Similarly, Callagy et al. [12] successfully used TMA immunohistochemistry for emulating molecular classification of breast cancer. TMAs may also be used for in situ hybridization studies [13].

Several choices have to be made before starting a TMA experiment. A principal one is the size of the array itself and the diameter of individual cores. The core size may range from 0.5 to $3 \mathrm{~mm}$ and $0.6 \mathrm{~mm}$ was applied in our study; while the arrays with smallest cores contain hundreds of cases, the ones with largest cores will consist of a few dozen samples only.

Despite its obvious advantages TMA technology suffers from some drawbacks. The principal one is the limited size of the core examined. A single core contains only a small fraction of the entire section which, by itself, represents only a small fraction of an 

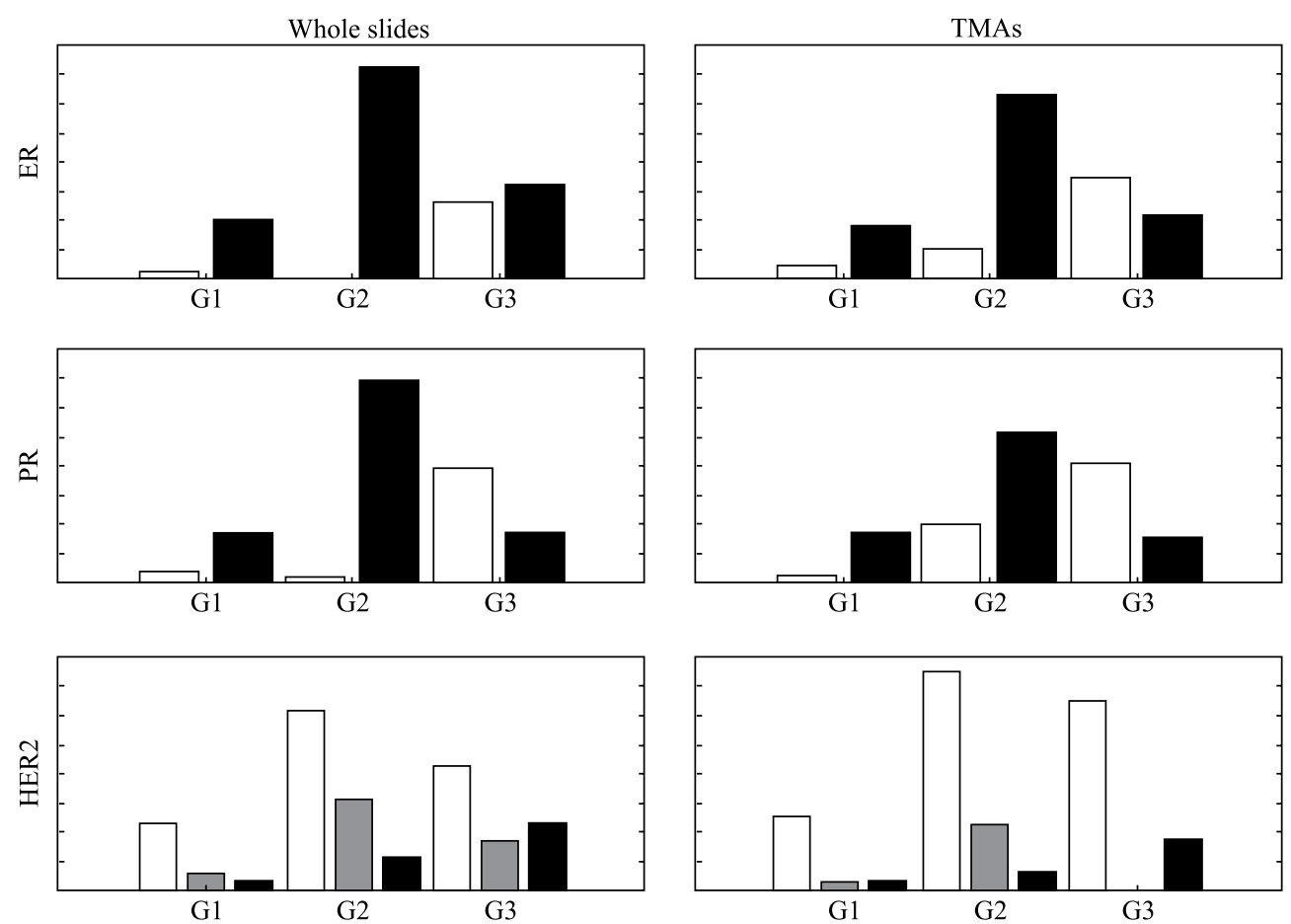

$\square$ Negative $\square$ Ambigous (for HER2) $\square$ Positive

Figure 6. The relationship between tumor grade and receptors expression. Whole slides (left) and TMAs (right) are shown in the same scale

Table 4. HER2 receptor positive and negative results assessed by two methods

\begin{tabular}{|l|l|l|l|}
\hline \multicolumn{2}{|c|}{} & \multicolumn{2}{|l|}{ TMA } \\
\cline { 2 - 4 } \multicolumn{2}{|c|}{} & Ambiguous* & Positive \\
\hline \multirow{2}{*}{$\begin{array}{l}\text { Whole } \\
\text { slides }\end{array}$} & Negative & 2 & 0 \\
\cline { 2 - 4 } & Ambiguous* & 9 & 0 \\
\cline { 2 - 4 } & Positive & 1 & 9 \\
\hline Positive predictive value & 1.00 \\
\hline Negative predictive value & 0.73 \\
\hline Sensitivity & 0.69 \\
\hline Specificity & 0.96 \\
\hline Kappa & 0.55 \\
\hline
\end{tabular}

* $1+$ result, requiring FISH for therapy planning

entire lesion. Thus, TMAs are most useful for detecting markers with a diffuse and uniform expression within a lesion. Although this technique may be less adequate for analyzing parameters which, by their nature, vary between various tumor tissue regions differing in microvessel density or tumor-related inflammatory infiltrate, we used it successfully for microvessel analysis [14]. Early and in situ lesions may be particularly difficult to be properly included in the TMAs [15]. Further progress may be obtained with TMA technology via integration with imaging systems, allowing computer-aided array design and scoring based on image-analysis. With this methodology, the possibility arises to choose cores from specific compartments, e.g. inside of the lesion, infiltration front, perilesional inflammatory infiltrate, etc. [16]. However, by using such approach, non-trivial problems will be encountered like separation of neoplastic from non-neoplastic cells, exclusion of poorly preserved tissue, and overlapping nuclei. Some of these problems may be solved using semiautomatic, human-manned systems. Unfortunately, this will limit the profits of automated analysis. Faratian et al. [17] have validated the uses of image analysis system for the evaluation of steroid receptors in breast cancer; they found that a sufficient accuracy may be obtained with just two cores and an excellent accuracy with six cores. Additionally, the use of virtual slides technology, like in the present study, may help the manual assessment. Another drawback of the TMA technique is the tissue core loss. Inevitably, even in experienced hands, some tissue cores may contain no tumor and others may be positioned outside the plane of section. Furthermore, during the staining procedure, especially during antigen retrieval, some of the cores are lost. To limit the influence of tissue loss the usual procedure is to include several cores from a single case which limits the advantages of the technology. On the other hand, 
including several cores makes TMA more representative of the entire case.

Although the TMAs have been used for more than 15 years, the optimal number of cores has yet not been standardized, and various authors may propose different solutions. E.g. Camp et al. [18] validated the use of TMAs for BC. They demonstrated that just two $0.6 \mathrm{~mm}$ TMA cores are sufficient for proper assessment of hormonal and HER2 receptors. Zang et al. [19] analyzed the results of TMA assessment of HER2 in breast cancer; they used only one 0.6 core per case and reported high accuracy of the method. Highest discordance rates were seen for $2+$ staining cases. According to these authors $2+$ cases should be reevaluated using immunohistochemistry and FISH on whole slides [19]. Graham et al. [20] also advocated for the practical application of TMA for HER2 evaluation in BC, yet reported that 5 cores were needed for proper assessment of the marker and obtained extremely good ( $>95 \%)$ agreement between whole slides and TMAs. Kyndi et al. [21] analyzed the problem of staining heterogeneity encountered by including TMA cores from different paraffin blocks and different parts of the block. They reported very significant concordance of the results for a single breast tumor and concluded that a single core was sufficient for consistent evaluation. Conversely, Lin et al. [7] concluded that TMA concordance with standard immunohistochemistry strongly depended on the number of cores studied. The ranges of specificity and sensitivity seen by Lin et al. were similar to those found in our study. Lin et al. concluded that TMA should be used for biomarker research, however caution is advised when applying this technology for the purposes of diagnostic pathology. On a small series of cases, Awadelkarim et al. [5] by comparing TMAs with classical IHC evaluation reported for TMAs sensitivities of $77.8 \%, 82.8 \%, 83.3 \%$, and negative predictive values of $64.7 \%, 72.2 \%, 96.4 \%$ for ER, PR and HER2, respectively. Their results were thus similar to ours but no relationship was found between the tumor size and TMA accuracy in our material.

TMAs were also extensively used for quality assurance and control, facilitating the process of the same staining on several tissues, allowing for the identification of discrepancies. Thus, in Wasielewski et al. study [22] tumors with more ambiguous staining results tended to show higher inter-observer and inter-laboratory variability. Diaz et al. [23] specifically tested the feasibility of TMAs for validation of HER2 FISH test. In this setting, the tissue microarrays were stained in different laboratories and the results were compared. These authors reported a perfect concordance in all the informative cases. Programs of quality control on TMAs have been also introduced in Poland for the validation of HER2 FISH test (External Quality Assessment Scheme, run by Abbott Molecular).

In conclusion, we showed that tissue microarray technology may provide results comparable to whole sections in assessing receptor immunohistochemistry of breast carcinoma. The positive results were highly consistent, yet negative predictive value was not entirely satisfactory. Nevertheless, the relationship between clinicopathologic data and immunohistochemistry performed on whole slides and TMAs were found to be fairy similar.

\section{Acknowledgements}

The authors thank Mr. Ian B. Perera for help in revising the manuscript.

\section{References:}

1. Gudlaugsson E, Klos J, Skaland I et al. Prognostic comparison of the proliferation markers mitotic activity index, phosphohistone H3, Ki67, steroid receptors, HER2, high molecular weight cytokeratins and classical prognostic factors in T12N0M0 breast cancer. Pol J Pathol. 2013;64:1-8.

2. Adamczyk A, Niemiec J, Ambicka A et al. Expression of ER/ PR/HER2, basal markers and adhesion molecules in primary breast cancer and in lymph nodes metastases: a comparative immunohistochemical analysis. PolJ Pathol. 2012;63:228-234.

3. Kowalik A, Kopczynski J, Wypiorkiewicz E et al. Active transport of RB protein from the nucleus to the cytoplasm as one of the development mechanisms of HER2-positive breast cancer. Pol J Pathol. 2013;64:9-14.

4. Dekker TJ, Borg ST, Hooijer GK et al. Determining sensitivity and specificity of HER2 testing in breast cancer using a tissue micro-array approach. Breast Cancer Res. 2012;14: R93.

5. Awadelkarim KD, Arizzi C, Elamin EO et al. Tissue microarray (TMA) versus whole section immunohistochemistry in the assessment of ER/PR and Her-2/neu status in a breast cancer series from Sudan. Breast J. 2013;10.1111/tbj.12144.

6. Batistatou A, Televantou D, Bobos Met al. Evaluation of current prognostic and predictive markers in breast cancer: a validation study of tissue microarrays. Anticancer Res. 2013;33: 2139-2145.

7. Lin $\mathrm{Y}$, Hatem $\mathrm{J}$, Wang $\mathrm{J}$ et al. Tissue microarray-based immunohistochemical study can significantly underestimate the expression of HER2 and progesterone receptor in ductal carcinoma in situ of the breast. Biotech Histochem. 2011;86:345-350.

8. Wolff AC, Hammond ME, Schwartz JN et al. American Society of Clinical Oncology/College of American Pathologists guideline recommendations for human epidermal growth factor receptor 2 testing in breast cancer. J Clin Oncol. 2007;25:118-145.

9. Battifora H. The multitumor (sausage) tissue block - novel method for immunohistochemical antibody testing. Lab Invest. 1986;55:244-248.

10. Kononen J, Bubendorf L, Kallioniemi A et al. Tissue microarrays for high-throughput molecular profiling of tumor specimens. Nat Med. 1998;4:844-847.

11. Tamimi RM, Baer HJ, Marotti J et al. Comparison of molecular phenotypes of ductal carcinoma in situ and invasive breast cancer. Breast Cancer Res. 2008;10:R67. 
12. Callagy G, Cattaneo E, Daigo Y et al. Molecular classification of breast carcinomas using tissue microarrays. Diagn $\mathrm{Mol}$ Pathol. 2003;12:27-34.

13. Okon K, Sinczak-Kuta A, Klimkowska A et al. Tissue microarray FISH applied to colorectal carcinomas with various microsatellite status. Pol J Pathol. 2006;57:99-103.

14. Okon K, Kawa R. Microvascular network in renal carcinomas. Quantitative and tissue microarray immunohistochemical study. Pol J Pathol. 2008;59:107-115.

15. Camp RL, Neumeister V, Rimm DL. A decade of tissue microarrays: progress in the discovery and validation of cancer biomarkers. J Clin Oncol. 2008;26:5630-5637.

16. Zlobec I, Koelzer VH, Dawson $\mathrm{H}$ et al. Next-generation tissue microarray (ngTMA) increases the quality of biomarker studies: an example using CD3, CD8, and CD45RO in the tumor microenvironment of six different solid tumor types. J Transl Med. 2013;11:104.

17. Faratian D, Kay C, Robson T et al. Automated image analysis for high-throughput quantitative detection of ER and PR expression levels in large-scale clinical studies: the TEAM Trial Experience. Histopathology. 2009;55:587-593.
18. Camp RL, Charette LA, Rimm DL. Validation of tissue microarray technology in breast carcinoma. Lab Invest. 2000;80:1943-1949.

19. Zhang D, Salto-Tellez M, Do E et al. Evaluation of HER-2/ /neu oncogene status in breast tumors on tissue microarrays. Hum Pathol. 2003;34:362-368.

20. Graham AD, Faratian D, Rae F et al. Tissue microarray technology in the routine assessment of HER-2 status in invasive breast cancer: a prospective study of the use of immunohistochemistry and fluorescence in situ hybridization. Histopathology. 2008;52:847-855.

21. Kyndi M, Sorensen FB, Knudsen $\mathrm{H}$ et al. Tissue microarrays compared with whole sections and biochemical analyses. A subgroup analysis of DBCG 82 b\&c. Acta Oncol. 2008;47:591-599.

22. Wasielewski R, Hasselmann S, Ruschoff J et al. Proficiency testing of immunohistochemical biomarker assays in breast cancer. Virchows Arch. 2008;453:537-543.

23. Diaz LK, Gupta R, Kidwai N et al. The use of TMA for interlaboratory validation of FISH testing for detection of HER2 gene amplification in breast cancer. $J$ Histochem Cytochem. 2004;52:501-507.

Submitted: 10 July, 2013

Accepted after reviews: 6 January, 2014 